\title{
A Broadband Millimeter-Wave Waveguide Power Divider with High Isolation
}

\author{
Hua Zhang*, Deng Yun Shao, Sheng Zeng \\ Nanjing Electronic Devices Institute, Nanjing, China \\ Email address: \\ emfield_hz@126.com (Hua Zhang) \\ ${ }^{*}$ Corresponding author
}

To cite this article:

Hua Zhang, Deng Yun Shao, Sheng Zeng. A Broadband Millimeter-Wave Waveguide Power Divider with High Isolation. American Journal of Physics and Applications. Vol. 7, No. 4, 2019, pp. 101-108. doi: 10.11648/j.ajpa.20190704.12

Received: May 27, 2019; Accepted: July 16, 2019; Published: August 5, 2019

\begin{abstract}
In this paper, an $E$-plane stepped-impedance transformer and Y-junction bifurcation are used to form a waveguide power divider with ceramic substrate loaded with thin film resistors. This structure is realized high isolation in $V$-band by inserting a ceramic substrate at the $H$-plane center of the Y-junction waveguide bifurcation, both sides of which loaded with thin film resistors. The waveguide power divider was fabricated with aluminium-50\% silicon, and has characteristics of light weight, lower coefficient of thermal expansion, good thermal conductivity, and its properties are more compatible with those of ceramic substrate. The principle and design procedure are described in detail. A $V$-band $E$-plane waveguide power divider is designed, fabricated, and measured. The measured results show that insertion loss is less than $0.4 \mathrm{~dB}$ in the frequency range of $50 \sim 60 \mathrm{GHz}$, with typical isolation levels of $25 \mathrm{~dB}$ between the two output ports and amplitude imbalance less than $0.19 \mathrm{~dB}$, phase imbalance less than $1.4^{\circ}$. The measured and simulated results show good amplitude, phase, and isolation characteristics validating the proposed power divider.
\end{abstract}

Keywords: Millimeter-wave Waveguide Power Divider, High Isolation, Wide Bandwidth, Aluminium-50\% Silicon

\section{Introduction}

Waveguide power divider is an important component commonly used in microwave and millimeter-wave system, which be widely used in power dividers/combiners, antenna feed systems and so on. In a power divider structure, when power is fed from an input port, the presence of other paths introduces reflection, which requires a matching structure to counteract the reflection. For example, $E$-plane stepped-ladder matching power divider, $E$-plane T-junction series power divider, $H$-plane T-junction power divider with metal reflector matching, $H$-plane T-junction power divider with inductive cylinder matching, etc [1-2]. The machining $H$-plane waveguide power divider is split from the $H$-plane. The junction surface of the waveguide cavity is perpendicular to the wall current direction and the current path is cut off. Because millimeter-wave wavelength is short, the small slot at the junction of waveguide cavity will lead to the deterioration of waveguide performance. The machining and assembly accuracy of waveguide cavity is required to be very high.
$E$-plane waveguide power divider is split into two blocks from the center of wide side and the current path isn't cut off. Therefore, the radiation-free assembly of waveguide cavity can be realized. It has no effect on the distribution of electromagnetic field in the waveguide, and the performance is stable and reliable. However, the traditional $E$-plane waveguide power divider lacks isolation resistance, and there is mutual interference between the paths of power divider. In general, in the millimeter-wave band, in order to realize high output isolation, magic-T structures or resistive films are applied as absorbing loads. Wang and Zheng describe a waveguide power divider with good isolation was realized by using the $E$-plane folded magic-T structure [3], but the size is large and the structure is complex. Guo et al. propose a compact $X$-band waveguide magic-T which is realized by the $E$-plane power divider and ridge waveguide coupling structure for high-power solid-state power combining [4], and the isolation between the sum/difference ports and the two input port is better than 40 and $26 \mathrm{~dB}$, but the complexity of the combination increase. Guo et al. also propose two kinds of four-way power combiners with high degree of 
isolation [5]. Takeda and Epp et al. investigate the resistive septum loaded was inserted in the center of the $H$-plane of main transmission waveguide to improve the isolation between the ports $[6,7]$. Chen demonstrates the resistive slots and the mode suppressing resistive cards were used in the power divider design provided an average of $30 \mathrm{~dB}$ isolation among the output ports, except for the adjacent port isolation of $20 \mathrm{~dB}$ [8]. Dong et al. present two broadband E-plane in-phase and out-of-phase waveguide power dividers with high isolation based on the substrate-integrated waveguide (SIW) T-junction with isolation resistor and SIW-to-waveguide transition circuit [9]. Although the dividers can be used in the whole $\mathrm{Ka}$-band, but its isolation, amplitude and phase balance are greatly influenced by SIW T-junction and SIW-to-waveguide transition circuit behavior, and the design and fabricating process is complex. Song et al. design a $K a$-band rectangular waveguide Gysel power divider with greater than $15 \mathrm{~dB}$ output isolation [10]. The H-plane waveguide power divider has two E-plane T-junctions, which are connected to the matched load of the waveguide to form the isolation between the output ports. The millimeter-wave absorbing materials are used to fill two matched loads. Ding et al. propose a five-port waveguide power divider with higher than $18 \mathrm{~dB}$ isolation [11]. Chu et al. design an $E$-plane magic-T with coplanar arms with $20 \mathrm{~dB}$ isolation. However, the waveguide-to-microstrip transition restricts its power capacity to several watts [12].

In this paper, an E-plane Y-junction waveguide power divider made of aluminium- $50 \%$ silicon is designed. A ceramic substrate loaded with thin film resistors is inserted in the center of the $H$-plane of the Y-junction bifurcation waveguide for broadband and good isolation. Furthermore, the highly symmetric of the proposed power divider provides good amplitude and phase balance between the two output ports. A $V$-band $E$-plane waveguide power divider is designed, fabricated, and measured. The measured results are in good agreement with the simulated ones. This paper is described as follows: Firstly, the design of waveguide power divider is analyzed theoretically. Secondly, the numerical simulation and optimization are carried out. Finally, the measured results and their analysis are given.

\section{Theory}

The $E$-plane waveguide power divider is composed of an $E$-plane stepped-impedance transformer with two $E$-plane branches connected behind it. This type of power divider has the following advantages over other waveguide power dividers. Firstly, the waveguide wall of separated output port is perpendicular to the electric field of main mode for rectangular waveguide. Therefore, the introduced electromagnetic field distortion is limited. Secondly, the broadband power divider can be obtained with relatively few number of transform segments. Thirdly, in terms of volume and weight, it is the most effective for $E$-plane distribution of rectangular waveguides, which is suitable for $E$-plane block assembly.
For the given operating bandwidth of the power divider: 50 to $60 \mathrm{GHz}$, the input reflection loss is required not to be greater than $-20 \mathrm{~dB}$. Since our design is in the $V$ band, a standard waveguide WR-15 ( $a=3.76 \mathrm{~mm}, b=1.88 \mathrm{~mm})$ is used. All the ports are standard waveguide ports. The height of the input and output waveguide is $b=1.88 \mathrm{~mm}$, and the thickness of the isolation wall is $t=0.25 \mathrm{~mm}$. In order to obtain a wider bandwidth, a multi-step impedance matching structure is used to design the stepped-impedance transformer, and the number of transform segments $\mathrm{N}$ can be obtained to be 3 , and there are $N+1=4$ steps [1], as shown in Figure 1, where $b_{4}=2 * b+t$.

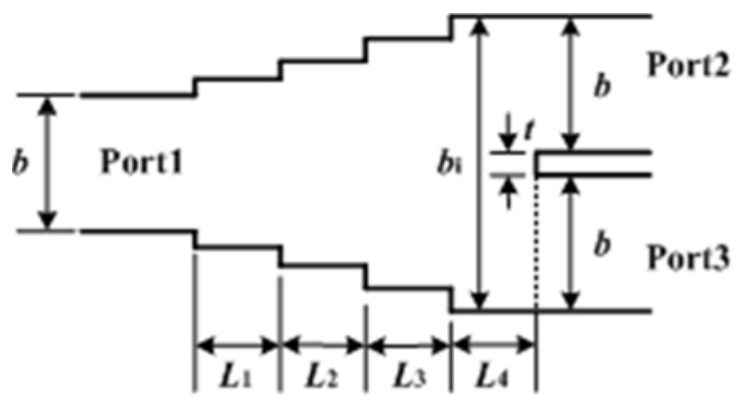

Figure 1. E-plane waveguide power divider with stepped-impedance transformer.

The voltage standing wave radio $\rho_{\mathrm{i}}(i=1 \sim N+1)$ of the corresponding junction in the section of the stepped-impedance transformers can be calculated according to the principle of $1 / 4$ wavelength transformer. The characteristic impedance $Z_{i}$ is proportional to the height of the waveguide $b_{i}$, as shown in (1):

$$
\rho_{\mathrm{i}}=\frac{\mathrm{Z}_{i}}{\mathrm{Z}_{i-1}}=\frac{Y_{i-1}}{Y_{i}}=\frac{b_{i}}{b_{i-1}}
$$

Where, $Y_{\mathrm{i}}$ is the characteristic admittance.

The normalized junction admittance is:

$$
\frac{B_{i}}{Y_{i-1}}=\frac{\left(1-S_{11}\right)\left(1-S_{22}\right)-S_{12} S_{21}}{2 j S_{21}}
$$

In (2), $B_{i}$ is the junction admittance and $S_{11}, S_{12}, S_{21}, S_{22}$ are the scattering parameters of the scattering matrix. The length of the transformer segment $L_{i}$ is:

$$
L_{i}=\frac{\lambda_{g 0}}{2 \pi}\left[\frac{\pi}{2}+x_{i}-X_{i+1}\right]
$$

Where $\lambda_{\mathrm{g} 0}$ is the guiding wavelength of the central frequency and $x_{i}$ and $X_{i}$ are given by Matthaei et al. [13]:

$$
x_{i}=\frac{1}{2}\left\{\arctan \left[\frac{\frac{B_{i}}{Y_{i-1}}}{1-\frac{1}{\rho_{i}}}\right]-\arctan \left[\frac{\frac{B_{i}}{Y_{i-1}}}{1+\frac{1}{\rho_{i}}}\right]\right\}
$$




$$
X_{i}=\frac{1}{2}\left\{\arctan \left[\frac{\frac{B_{i}}{Y_{i-1}}}{1-\frac{1}{\rho_{i}}}\right]+\arctan \left[\frac{\frac{B_{i}}{Y_{i-1}}}{1+\frac{1}{\rho_{i}}}\right]\right\}
$$

The normalized junction admittance is calculated by mode-matching method at the center frequency, which contains the $T E_{1 n}^{x}$ modes caused by the corresponding discontinuities. The stepped-impedance transformer sections designed in this paper is a rectangular waveguide with the discontinuity of $E$-plane both sides, as shown in Figure 2. By using the mode-matching method [14], the generalized scattering matrix of the discontinuous interface of the basic element is obtained, and the length of the impedance transformer section $L_{i}(i=1 \sim N+1)$ can be obtained by using (1) (4).

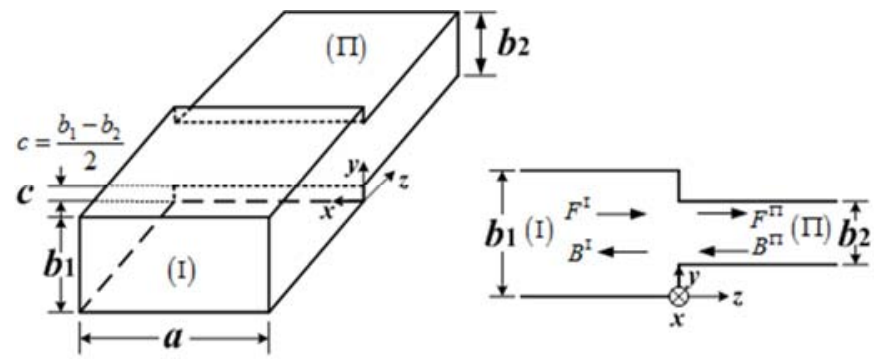

Figure 2. Rectangular waveguide element with stepped discontinuity of E-plane both sides.
Mode matching method is a numerical method based on electromagnetic field theory, which is based on the approximate expansion of unknown electromagnetic field components by orthogonal series, matching on the discontinuous interface according to the tangential field component equality, and finally obtaining the generalized scattering matrix. Figure 2 shows rectangular waveguide element with stepped discontinuity of $E$-plane both sides. As shown in Figure 2, the stepped interface is taken at $z=0$, and when the main mode $T E_{10}$ is excited, a high-order mode will be generated at $z=0$, and the field components of $E_{z} 、 H_{y}$ will be generated in addition to the incident field components of $E_{y}$ 、 $H_{x} 、 H_{z}$, so the electromagnetic field has the five components in the stepped discontinuity.

$$
\left\{\begin{array}{c}
\vec{E}=\nabla \times \vec{A}_{h} \\
\vec{H}=\frac{j}{\omega \mu_{0}} \nabla \times \nabla \times \vec{A}_{h}
\end{array}\right.
$$

Equation (5) is used to represent the electromagnetic field in the discontinuous region, these field components can be derived from the $x$ component of vector potential: $\vec{A}_{h}=A_{h x} \vec{e}_{x}$. Because the structure does not change in the $x$ direction, the electromagnetic field only needs to be approximated in the $y$ direction. The mode ( $T E_{1 n}^{x}$ mode) for extracting from I and $\Pi$ regions is:

$$
\begin{gathered}
A_{h x}^{\mathrm{I}}=\sum_{n=0}^{N} G_{n}^{\mathrm{I}} \sin \left(\frac{\pi}{a} x\right) \frac{\cos \left(\frac{n \pi}{b_{1}} y\right)}{\sqrt{1+\delta_{0 n}}}\left[F_{n}^{\mathrm{I}} e^{-j k_{z n}^{\mathrm{I}} z}-B_{n}^{\mathrm{I}} e^{+j k_{z n}^{\mathrm{I}} z}\right] \\
A_{h x}^{\Pi}=\sum_{m=0}^{M} G_{m}^{\Pi} \sin \left(\frac{\pi}{a} x\right) \frac{\cos \left(\frac{m \pi}{b_{2}}\left(y-\frac{b_{1}-b_{2}}{2}\right)\right)}{\sqrt{1+\delta_{0 m}}}\left[F_{m}^{\Pi} e^{-j k_{z m}^{\Pi} z}-B_{m}^{\Pi} e^{+j k_{z m}^{\Pi} z}\right]
\end{gathered}
$$

In (6), $m$ and $n$ are modular ordinal numbers, respectively. Both $z$ in $k_{z n}^{\mathrm{I}}$ and $k_{z m}^{\Pi}$ subscript and $z$ in $e^{z}$ superscript indicate the direction of coordinate $z$ propagation, as shown in Figure 2. And $\delta_{0 n}$ and $\delta_{0 m}$ are Kronecker $\delta$ functions, $k_{z n}^{\mathrm{I}}$ and $k_{z m}^{\Pi}$ are propagation constants, $G_{n}^{\mathrm{I}}$ and $G_{m}^{\Pi}$ are power normalization coefficients. The modes of each region are follows: $N$ modes are taken on both sides of the stepped discontinuity. $F$ and $B$ are the amplitudes of incident and reflected waves, respectively. Two necessary conditions for field components matching are used in the discontinuity $(z=0)$ :

$$
\begin{aligned}
& \left\{\begin{array}{c}
E_{y}^{\mathrm{I}}=0 \quad 0<y<c, b_{1}-c<y<b_{1} \\
E_{y}^{\mathrm{I}}=E_{y}^{\Pi} \quad c<y<b_{1}-c
\end{array}\right. \\
& \text { And } H_{x}^{\mathrm{I}}=H_{x}^{\Pi} \quad c<y<b_{1}-c
\end{aligned}
$$

By using the orthogonality among the modes, the relationship between the incidence and reflection coefficient can be obtained, and then the scattering matrix at discontinuity of $E$-plane both sides can be obtained.

\section{Design and Analysis}

\subsection{Realization of E-plane Y-junction Bifurcation Waveguide Power Divider}

The stepped-impedance transformer sections of $E$-plane waveguide power divider can be decomposed into four stepped elements of $E$-planes both sides shown in Figure 2. According to the above analysis, the initial values of the structural parameters $\left(b_{1} \sim b_{3}\right.$ and $\left.L_{1} \sim L_{4}\right)$ of $E$-plane waveguide power divider shown in Figure 1 can be calculated by programming with (1) (7).

In order to facilitate the assembly and test of the output port, 
each output port of the power divider is symmetrically connected with two $90^{\circ}$ circular arc waveguide bend structures (radii of $r_{1}$ and $r_{2}$, respectively) to form a Y-junction bifurcation structure. This ensures that the input and output ports are in the same direction, as shown in Figure 3. Considering the actual machining, the circular arc transition is designed at the step, and its contour matches with the outer edge of the milling cutter, which can be easily formed by milling machine.

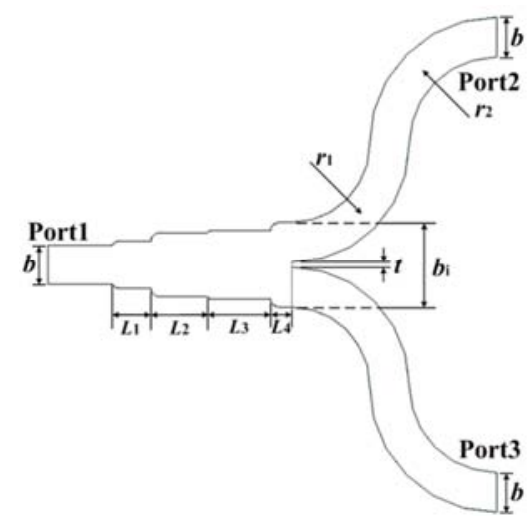

Figure 3. Top view of the proposed E-plane Y-junction bifurcation waveguide power divider.

Table 1. Dimensions of E-plane Y-Junction bifurcation Waveguide Power Divider (Unit: $\mathrm{mm}$ ).

\begin{tabular}{ll}
\hline Parameters & Optimized Values \\
\hline$b_{1}$ & 2.4 \\
$b_{2}$ & 3.3 \\
$b_{3}$ & 3.5 \\
$b_{4}$ & 3.76 \\
$L_{1}$ & 1.9 \\
$L_{2}$ & 2.4 \\
$L_{3}$ & 2.6 \\
$L_{4}$ & 1.8 \\
$r_{1}$ & 4.5 \\
$r_{2}$ & 4.5 \\
\hline
\end{tabular}

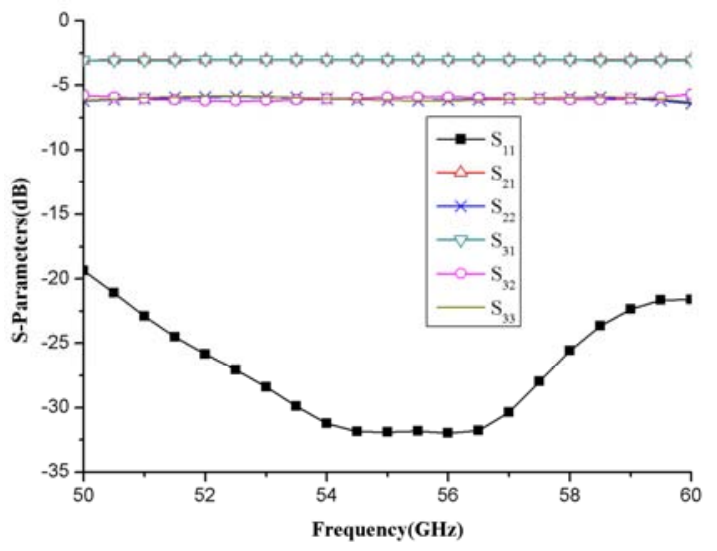

Figure 4. Simulated scattering parameters of E-plane Y-junction bifurcation waveguide power divider.

With the help of 3D full-wave electromagnetic simulation software HFSS, E-plane Y-junction bifurcation waveguide power divider was simulated and optimized, and the optimum structure dimensions is shown in Table 1. Figure 4 shows the optimized reflection and transmission characteristic simulated curve. According to microwave network theory [15], the lossless reciprocal three-port network can not realize the matching of three ports at the same time. If the port reflection and the isolation between the ports are compromised, the minimum $S_{11}$ is taken as the design goal. Through the analysis, it can be seen that the typical return loss of the mismatched port is $6 \mathrm{~dB}$, and the isolation between the ports is about $6 \mathrm{~dB}$. This conclusion is also proved by the simulation results of $E$-plane waveguide power divider shown in Figure 4.

\subsection{Realization of E-plane Y-junction Waveguide Power Divider with High Isolation}

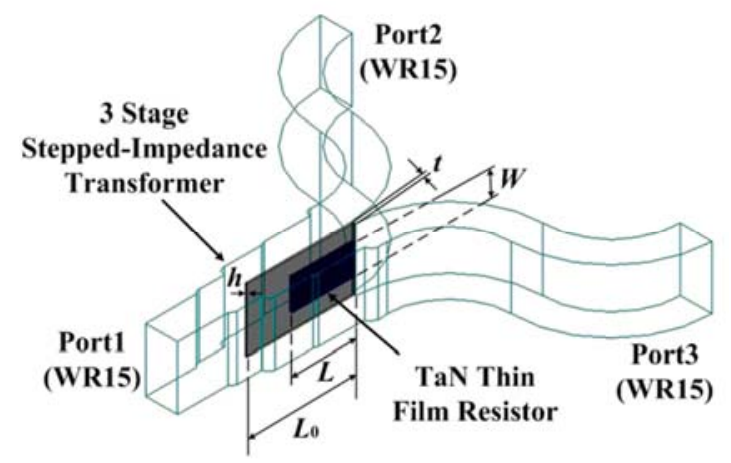

(a) E-plane Y-junction waveguide power divider with ceramic substrate loaded with thin film resistors

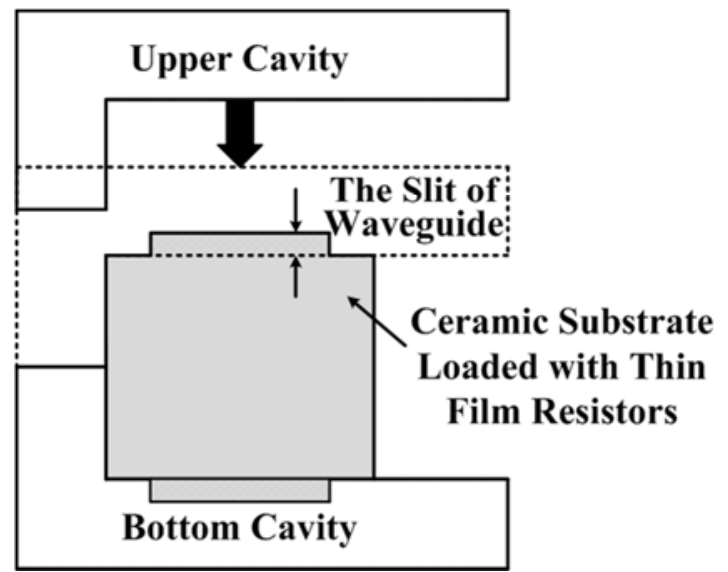

(b) Ceramic substrate loaded with thin film resistors inserted in the slit of waveguide H-plane

Figure 5. E-plane Y-junction waveguide power divider with ceramic substrate loaded with thin film resistors and the ceramic substrate is placed.

In this paper, an E-plane Y-junction bifurcation waveguide power divider with high isolation is designed. This structure compensates for the failure of the three-port device to match perfectly by inserting a ceramic substrate loaded with thin film resistors at the $H$-plane center of the Y-junction bifurcation waveguide of the main transmission waveguide shown in Figure 3. Thus, the problem of low isolation between the two output ports of the traditional $E$-plane Y-junction bifurcation power divider is solved, and the output standing wave ratio is improved. The insertion resistive loading substrate will 
increase insertion loss of waveguide power divider, considering the high frequency performance of millimeter-wave, and the insertion loss and the isolation between the two output ports of power divider are taken into account by partially loading resistive thin films. So as to uniformly reduce the effective area of the contact between the thin film resistor and the electromagnetic field, and reduces the insertion loss of power divider.

According to the high frequency loss characteristics of V-band millimeter-wave, the ceramic substrate is selected, the thickness of the substrate is $h=0.127 \mathrm{~mm}$, the material of thin film resistors are selected by TaN, the thickness of the material is $1 u \mathrm{~m}$, and the film resistors symmetrical map are coated on both sides of the ceramic substrate. Taking into account the process realizability of thin film resistors and the isolation requirements in the operating frequency band, the value of the thin film resistor was calculated to be $200 \Omega$ by HFSS,

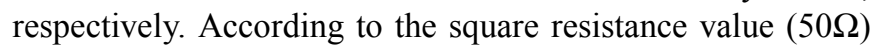
of the thin film resistor, the number $n$ of the required square resistance on each side of the ceramic substrate are calculated, and $n=4$ is obtained, from which the length $L$ and width $W$ of the thin film resistor can be obtained. The position where the thin film resistor is placed are shown in Figure 5 (a), in which the length of the ceramic substrate is $L_{0}$, the width is the dimension of the wide side of WR 15 waveguide: $3.76 \mathrm{~mm}$, and the whole structure is completely symmetrical.

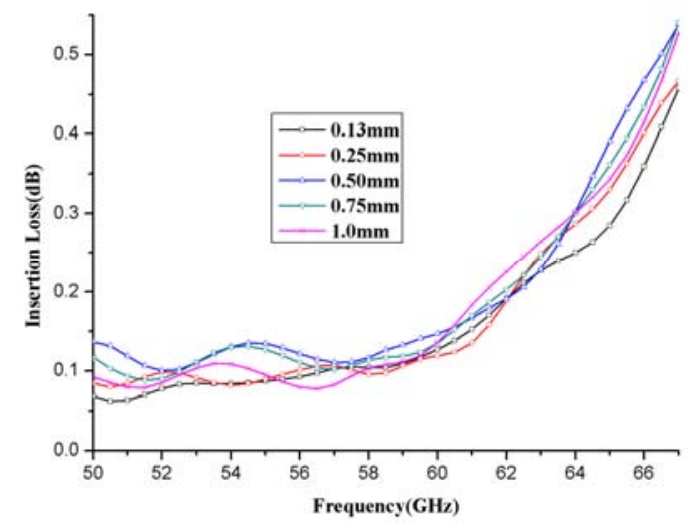

(a) Simulated insertion loss results with thickness t of the isolation wall

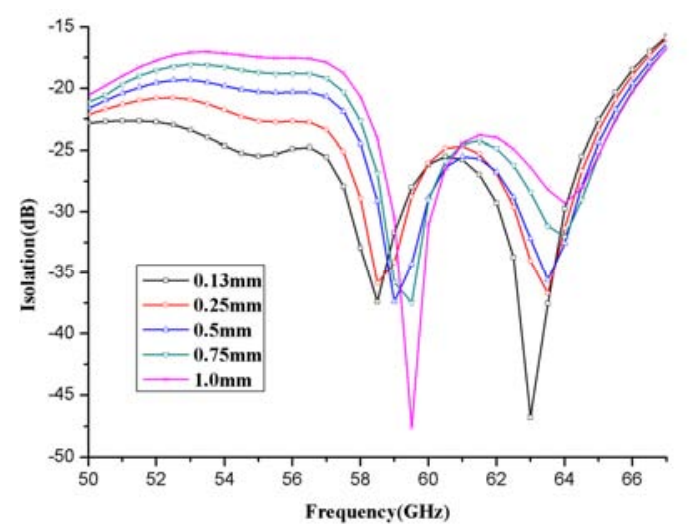

(b) Simulated isolation results with thickness t of the isolation wall

Figure 6. Simulated insertion loss and isolation with different thickness of the isolation wall.
The insertion of a resistive thin film ceramic substrate into the $E$-plane Y-junction bifurcation waveguide power divider shown in Figure 3 is bound to affect the electromagnetic field distribution of the entire structure. According to the requirements of the design target, the length $L_{0}$ of the ceramic substrate was simulated and optimized by HFSS. When the power divider is inserted into the ceramic substrate, the isolation is improved from $6 \mathrm{~dB}$ to about $25 \mathrm{~dB}$. By fine-tuning the height $\left(b_{1} \sim b_{3}\right)$ and length $\left(L_{1} \sim L_{4}\right)$ of the stepped-impedance transformer sections and the radius $\left(r_{1} \sim r_{2}\right)$ of the circular waveguide, a broadband matching can be achieved to meet the requirements of the design target. The thickness $t$ of the isolation wall also has an effect on the insertion loss and isolation of the power divider. The performance changing trend of the power divider when the value of $t$ varies from $0.13 \mathrm{~mm}$ to $1.0 \mathrm{~mm}$ is shown in Figure 6 . On the whole, when $t=0.13 \mathrm{~mm}$, the performance of the power divider is the best and becomes worse with the increase of thickness $t$. However, the thickness of $t=0.13 \mathrm{~mm}$ is too thin, so it is difficult to process it. Tradeoff between performance and machinability, $t=0.25 \mathrm{~mm}$ is more suitable.

Finally, the best structural dimensions parameters are obtained. Figure 7 shows the simulated electromagnetic field distribution of the E-plane Y-junction waveguide power divider. It can be seen that electromagnetic energy input by the waveguide is evenly distributed and output by the Y-junction bifurcation waveguide.

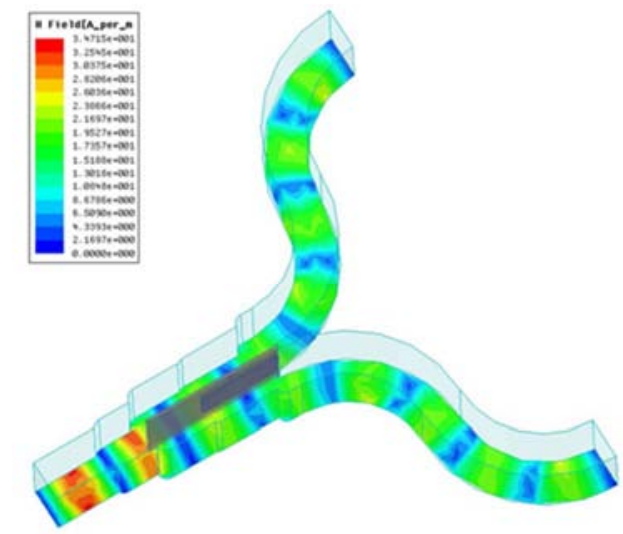

(a) Magnitude distribution of $\mathrm{H}$ field

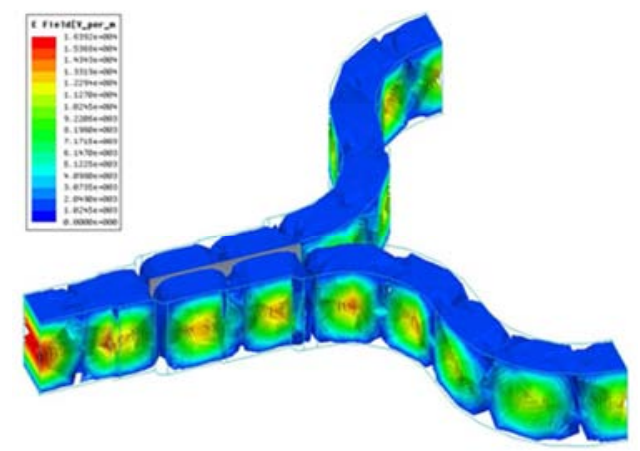

(b) Magnitude distribution of E field

Figure 7. Simulated field distributions of E-plane Y-junction waveguide power divider with ceramic substrate loaded with thin film resistors. 


\section{Fabrication and Measurement}

\subsection{Fabrication of Waveguide Power Divider}

Because ceramic substrate is loaded by the thin film resistors for isolation, in order to take into account the reliability requirements such as coefficient of thermal expansion matching, the waveguide power divider was fabricated with aluminium-50\% silicon instead of traditional brass H62 and plated with gold. Compared with brass H62, the material of aluminium- $50 \%$ silicon has the advantages of light weight, lower coefficient of thermal expansion, good thermal conductivity and so on. The material properties of aluminium-50\% silicon are more compatible with those of ceramic substrate $\left(99.5 \% \mathrm{Al}_{2} \mathrm{O}_{3}\right)$. The specific parameters are compared in Table 2 [16].

The power divider was split into two blocks (upper and bottom cavities) along the center line of the wide side of E-plane waveguide, the cavity was symmetrically spliced, and the ceramic substrate needs to be accurately assembled in the designed position. Therefore, silver epoxy bonding process was used for fabrication, and the fabricated structure is shown in Figure 5 (b).

Table 2. Comparison of Physical Characteristic of $99.5 \% \mathrm{Al} 2 \mathrm{O3}, \mathrm{Al}-50 \% \mathrm{Si}$ and $\mathrm{H} 62$.

\begin{tabular}{|c|c|c|c|c|}
\hline Materials & Density $\left(\mathrm{g} / \mathrm{cm}^{3}\right)$ & Coefficient of thermal expansion $\left(\mathrm{ppm} /{ }^{\circ} \mathrm{C}\right)$ & Tensile strength (MPa) & Thermal conductivity $(\mathrm{W} / \mathrm{m} \cdot \mathrm{K})$ \\
\hline $99.5 \% \mathrm{Al}_{2} \mathrm{O}_{3}$ & 3.9 & $7.5 \sim 8.3$ & 500 & 35 \\
\hline $\mathrm{Al}-50 \% \mathrm{Si}$ & 2.5 & 11.5 & 220 & 140 \\
\hline H62 & 8.5 & 19.3 & $\geq 294$ & 116 \\
\hline
\end{tabular}

In order to verify the performance of the designed power divider, a prototype of the proposed waveguide power divider was fabricated by the above process. The photograph of the power divider is shown in Figure 8 , and the overall size is: $41 \mathrm{~mm} \times 21 \mathrm{~mm} \times 20 \mathrm{~mm}$.

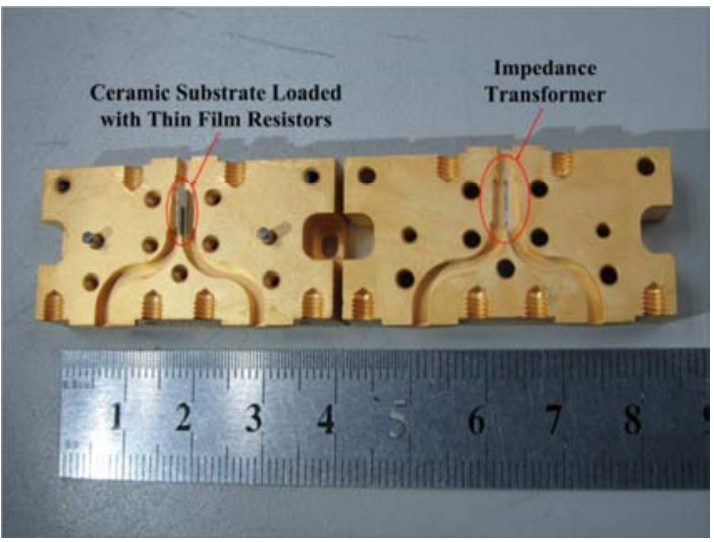

Figure 8. Photograph of the fabricated E-plane Y-junction waveguide power divider with ceramic substrate loaded with thin film resistor.

\subsection{Measured Results}

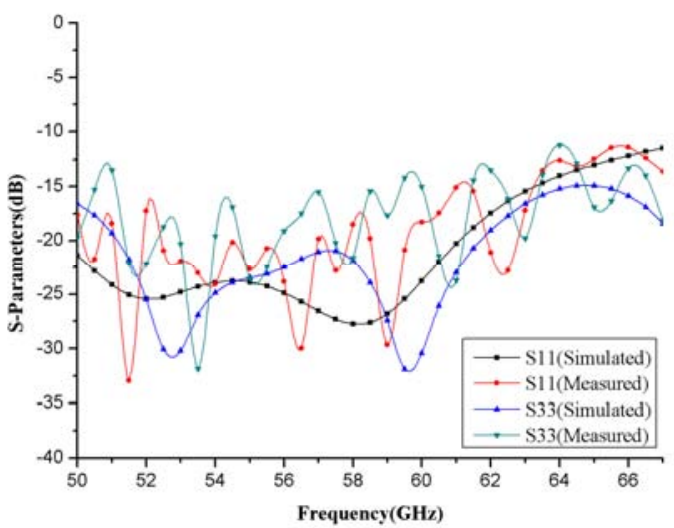

Figure 9. Simulated and measured results of the input and output return loss.

The waveguide power divider with ceramic substrate loaded with thin film resistor was measured by Agilent N5247A vector network analyzer (coaxial interface). The simulated and measured results of the input and output reflection characteristics, transmission and isolation characteristics of the power divider are shown in Figure 9 and Figure 10, respectively. It can be seen that the measured results agree well with the simulations. From 50 to $60 \mathrm{GHz}$, the measured return loss of input port is better than $16 \mathrm{~dB}$, the measured return loss of output ports are better than $13 \mathrm{~dB}$ (including return loss of coaxial to waveguide adapter for measuring), the measured insertion loss is less than $0.4 \mathrm{~dB}$, and the measured isolation between output ports is better than $18 \mathrm{~dB}$. Wherein, a frequency band of 54.5 to $64 \mathrm{GHz}$ for measured isolation more than $25 \mathrm{~dB}$ is realized. From the measured results, there is still good performance in the frequency range up to $67 \mathrm{GHz}$, which can be used in millimeter-wave circuits and systems. The difference of insertion loss between the two output ports is mainly due to the tolerance of the assembly position of the ceramic substrate loaded with thin film resistor. Compared with the simulation results, the measured performance is not good enough, mainly due to affected by the misalignment of the split blocks (that is, the degree of dislocation of the upper and bottom cavities) and the dimensions of the joints (depending on the smoothness of the joint between the upper and bottom cavities).

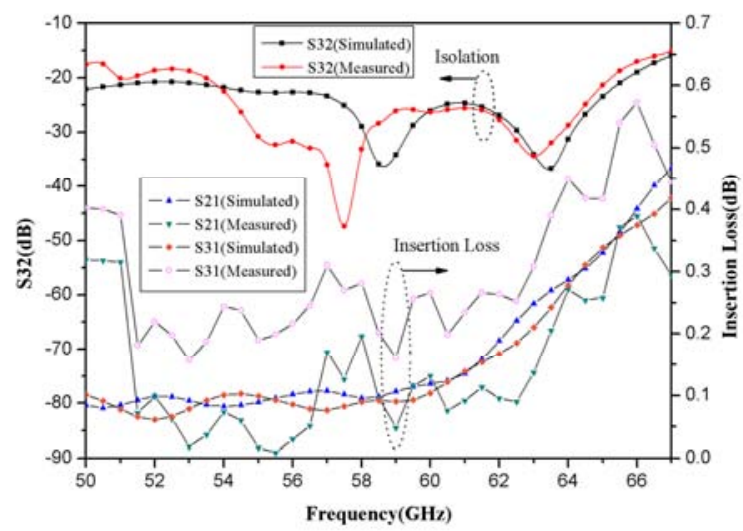

Figure 10. Simulated and measured results of the insertion loss and isolation. 


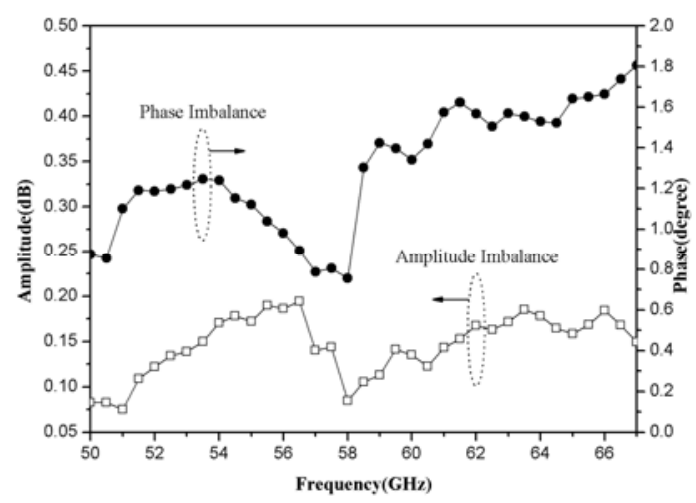

(a) Measured amplitude and phase imbalance between two output ports of the waveguide power divider

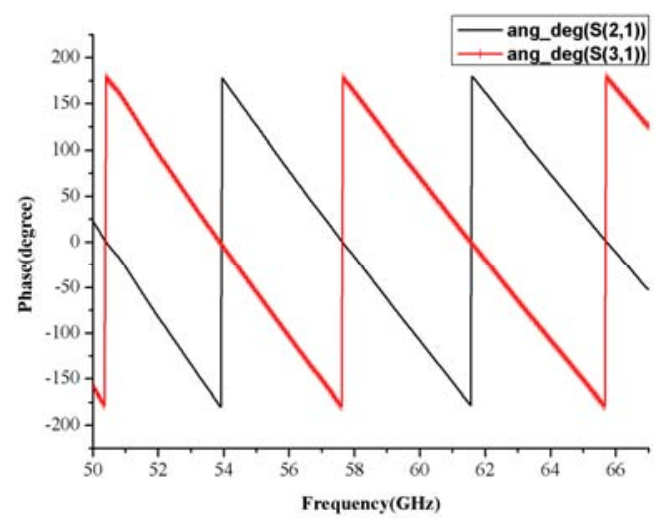

(b) Measured phase relation between two output ports of the waveguide power divider

Figure 11. Measured results of amplitude and phase imbalance between two output ports of the waveguide power divider.
Figure 11 shows the measured results of the amplitude and phase imbalance between two output ports of the waveguide power divider. It can be seen that the amplitude imbalance between two output ports is less than $0.19 \mathrm{~dB}$ in the $50 \sim 60 \mathrm{GHz}$ frequency range, the phase difference is $180^{\circ}$, and the phase imbalance is less than $1.4^{\circ}$ with the ideal difference of $180^{\circ}$. The waveguide power divider has excellent amplitude and phase consistency.

\subsection{Discussion Regarding Power-Handling Capability}

In order to analyze the power-handling capability of the proposed waveguide power divider, HFSS was used to simulate and analyze the electric field intensity distribution in the power divider, which is used to estimate the power-handling capability. When the calculated input power is $1 \mathrm{~W}$, the maximum electric field intensity inside the power divider are $1.64 \times 10^{4} \mathrm{~V} / \mathrm{m}$, which appear in the input port (port1), as shown in Figure 7 (b). According to the theoretical value $3 \times 10^{6} \mathrm{~V} / \mathrm{m}$ of air breakdown voltage under general conditions, the theoretical value of power-handling capability of the proposed waveguide power divider can reach $33 \mathrm{~kW}$. When the two output ports are mismatched or mismatched at the same time, and the reflected power of the two output ports is inconsistent, it can be observed that the maximum electric field intensity of the input port of the power divider does not change much, and the estimated value of power-handling capability of the power divider is basically unchanged.

Table 3. Comparison with some prior power divider.

\begin{tabular}{|c|c|c|c|c|c|c|}
\hline Reference & Frequency bandwidth & $\begin{array}{l}\text { Insertion loss } \\
\text { (dB) }\end{array}$ & $\begin{array}{l}\text { Isolation } \\
\text { (dB) }\end{array}$ & $\begin{array}{l}\text { Amplitude } \\
\text { imbalance (dB) }\end{array}$ & $\begin{array}{l}\text { Phase } \\
\text { imbalance }\left({ }^{\circ}\right)\end{array}$ & Structure \\
\hline [4] & $18 \%(7.8-9.4 \mathrm{GHz})$ & 0.2 & 26 & \pm 0.05 & 1 & Coaxial waveguide \\
\hline [5] work1 & $16 \%(7.6-8.9 \mathrm{GHz})$ & 0.2 & 21 & 0.1 & 1.5 & Coaxial waveguide \\
\hline [5] work2 & $14 \%(8-9.2 \mathrm{GHz})$ & 0.2 & 25 & 0.15 & 2 & Coaxial waveguide \\
\hline [9] & $40.6 \%(26.5-40 \mathrm{GHz})$ & $0.68-1.2$ & 15 & 0.4 & 3.5 & Waveguide-SIW \\
\hline [10] & $12.5 \%(30-34 \mathrm{GHz})$ & 0.25 & 15 & & & All-metal waveguide \\
\hline [11] & $12.1 \%(31-35 \mathrm{GHz})$ & 0.1 & 18 & \pm 0.1 & \pm 0.75 & All-metal waveguide \\
\hline [12] & $21 \%(27.5-34 \mathrm{GHz})$ & 0.35 & 20 & 0.25 & 2 & Waveguide-to-microstrip transition \\
\hline This work & $18.2 \%(50-60 \mathrm{GHz})$ & 0.4 & 25 & 0.19 & 1.4 & All-metal waveguide \\
\hline
\end{tabular}

\section{Conclusion}

In this paper, a novel configuration of a waveguide power divider with ceramic substrate loaded with thin film resistor has been proposed. This structure features combination of E-plane stepped-impedance transformer and Y-junction bifurcation, and the high isolation broadband waveguide power divider was realized, which was designed by using the full wave analysis method of electromagnetic field. A V-band waveguide power divider fabricated with aluminium-50\% silicon was present to verify this structure, and the radiation-free assembly of the waveguide power divider is realized. The measured results show that insertion loss is less than $0.4 \mathrm{~dB}$ in the frequency range of $50 \sim 60 \mathrm{GHz}$, with typical isolation levels of $25 \mathrm{~dB}$ between the two output ports and amplitude imbalance less than $0.19 \mathrm{~dB}$, phase imbalance less than $1.4^{\circ}$. Good agreement between the simulated and measured results has been observed. The theoretical value of power-handling capability of the waveguide power divider can reach $33 \mathrm{~kW}$. Moreover, the comparison of the presented power divider with other designs is summarized in Table 3 . The proposed power divider exhibits wide bandwidth, low insertion loss, high isolation, and light weight characteristics. These features qualify the proposed power divider for many applications in microwave and millimeter-wave systems, such as: radar, radiometer, communication. 


\section{References}

[1] J. Uher, J. Bornemann, and U. Rosenberg, Waveguide components for antenna feed systems: theory and CAD, Boston: Artech House, 1993, 104-252.

[2] J. Dittloff, J. Bornemann, and F. Arndt, "Computer aided design of optimum E- or H-plane N-furcated waveguide power dividers," IEEE $17^{\text {th }}$ European Microwave Conference, Rome. Italy, 1987, 10 (9), 181-186.

[3] Wang X, and Zheng T, "Design of high isolation Ka-band waveguide power divider with phase balance," IET International Radar Conference. Xi'an. China, 2013, 4.

[4] L. T. Guo, J. W. Li, W. H. Huang, H. Shao, T. Ba, T. Y. Jiang, Y. Jiang, and G. J. Deng, “ A waveguide magic-T coplanar arms for high-power solid-state power combining," IEEE Transactions on Microwave Theory and Techniques, 2017, 65 (8), 2942-2952.

[5] L. T. Guo, J. W. Li, W. H. Huang, H. Shao, T. Ba, T. Y. Jiang, Y. Jiang, and G. J. Deng, " Design of compact high-isolation four-way power combiners," IEEE Transactions on Microwave Theory and Techniques, 2018, 66 (5), 2185-2198.

[6] F. Takeda, O. Ishida, and Y. Isoda, "Waveguide power divider using metallic septum with resistive coupling slot," IEEE-MTTS International Microwave Symposium Digest. Dallas, American, 1982, 12, 527-528.

[7] Larry W. Epp, Daniel J. Hoppe, Abdur R. Khan and Scot L. Stride, "A high-power Ka-band (31-36GHz) solid-state amplifier based on low-loss corporate waveguide combing," IEEE Transactions on Microwave Theory and Techniques, 2008, 56 (8), 1899-1908.

[8] M. H. Chen, "A 19-way isolated power divider via the $\mathrm{TE}_{10}$ circular waveguide mode transition," IEEE-MTTS International Microwave Symposium Digest. Baltimore, Maryland, American, 1986, 6, 511-513.

[9] J. Dong, Y. Liu, Z. Q. Yang, and H. Peng, "Broadband millimeter-wave in-phase and out-of-phase waveguide dividers with high isolation," Journal of Infrared, Millimeter and Terahertz Waves, 2015, 36 (11), 1076-1088.
[10] K. J. Song, Y. C. Yan, Y. D. Zhou, M. Y. Fan, and Y. Fan, "Ka-band rectangular-waveguide Gysel power divider with low insertion loss and high output isolation," Journal of Infrared, Millimeter and Terahertz Waves, 2018, 39 (7), 996-1004.

[11] J. Y. Ding, Q. Y. Wang, and Y. B. Zhang, "A novel five-port waveguide power divider," IEEE Microwave \& wireless components letters, 2014, 24 (4), 224-226.

[12] Q. X. Chu, Q. S. Wu, and D. Y. Mo, "A Ka-band E-plane waveguide magic-T with coplanar arms," IEEE Transactions on Microwave Theory and Techniques, 2014, 62 (11), 2673-2679.

[13] George. L. Matthaei, L. Young, and E. M. T. Jones, Microwave filters, impedance-matching networks and coupling structures, Dedham Massachusetts: Artech House, 1980, 255-354.

[14] V. Zhurbenko, Passive microwave components and antennas, Croatia: InTech, 2010, 117-133.

[15] H. Kagan, "N-way power divider," IRE Transactions on Microwave Theory and Techniques, 1961, 9 (3), 198-199.

[16] Charles A. Harper, Electronic materials and processes handbook, 3rd ed., New York: McGraw-Hill Companies, Inc, 2004.

\section{Biography}

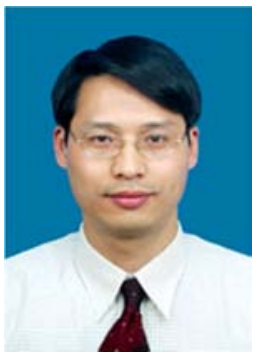

Hua Zhang received the M. S. degree in electrical engineering from Hefei University of Technology, HeFei, China, in 2000 and the $\mathrm{Ph}$. D. degree in electromagnetic theory \& microwave engineering from Southeast University, Nanjing, China, in 2005. He is currently working at Nanjing Electronic Devices Institute, Nanjing, China. His current research interests include developing active and passive circuits, antenna arrays for microwave and millimeter-wave, and development of millimeter-wave transceivers. He has authored or coauthored over 20 papers in journals and conferences. 